\title{
The extragalactic Cepheid bias: significant influence on the cosmic distance scale ${ }^{\star}$
}

\author{
G. Pature ${ }^{1}$ and P. Teerikorpi ${ }^{2}$ \\ 1 CRAL-Observatoire de Lyon, 69561 Saint-Genis Laval Cedex, France \\ e-mail: patu@obs.univ-lyon1.fr \\ 2 Tuorla Observatory, Turku University, Väisäläntie 20, SF21500 Piikkiö, SF, Finland \\ Received 8 October 2004 / Accepted 18 June 2005 \\ ABSTRACT \\ The unique measurements with the Hubble Space Telescope of Cepheid variable stars in nearby galaxies led to extragalactic distances that made \\ the HST Key Project conclude that the Hubble constant is $H_{0}=72 \mathrm{~km} \mathrm{~s}^{-1} \mathrm{Mpc}^{-1}$. The idea that $H_{0}$ is now known is widely spread among the \\ astronomical community. Some time ago, we suggested that a strong selection effect may still exist in the Cepheid method, resulting in too short \\ distances. Using a model similar to traditional bias corrections, we deduce here new estimates of distances from HST and previous ground-based \\ observations which are both affected by this effect, showing the same trend which starts at different distances. The recent measurement of M 83 \\ with the VLT is unbiased. Revisiting the calibration of HSTKP's with our new scale, makes long-range distance criteria more concordant and \\ reduces the value of $H_{0}$ to $\approx 60 \mathrm{~km} \mathrm{~s}^{-1} \mathrm{Mpc}^{-1}$. Locally, the corrected Cepheid distances give $H_{\text {local }}=56 \mathrm{~km} \mathrm{~s}^{-1} \mathrm{Mpc}^{-1}$ and reduce the velocity \\ dispersion in the Hubble flow. These numbers are indicative of the influence of the suggested Cepheid bias in the context of the HSTKP studies \\ and are not final values.
}

Key words. stars: variables: Cepheids - cosmology: distance scale

\section{Introduction}

By the 1970s, the value of the measured Hubble constant $H_{0}$ had changed from the old value of $530 \mathrm{~km} \mathrm{~s}^{-1} \mathrm{Mpc}^{-1}$ down to $50-100 \mathrm{~km} \mathrm{~s}^{-1} \mathrm{Mpc}^{-1}$, values of which were subject of debate (e.g., de Vaucouleurs 1984; Tammann 1996). The higher value was much lowered when it was understood that Malmquist-like biases for field galaxies and cluster galaxies result in distances that are grossly underestimated (Sandage \& Tammann 1975; Teerikorpi 1975). These biases have their roots in the cosmic dispersion of intrinsic properties of galaxies and the observational limits of samples. The mean luminosity of a "standard candle" is assumed to be constant, while at large distances only the brightest end of its cosmic distribution can be seen, because of the limiting magnitude imposed by the instrumentation (essentially, the size of the telescope and the sensitivity of the detector). If these biases are missed, this leads to underestimated distances and overestimated $H_{0}$.

The Hubble Space Telescope (HST) has provided astronomers with unprecedented high accuracy measurements free of atmospheric disturbance (Kennicutt et al. 1995). It can detect Cepheid stars in galaxies, up to the Virgo cluster. The determination of distances via the Period-Luminosity (PL) relation (Leavitt \& Pickering 1912; Madore \& Freedman 1991)

\footnotetext{
* Appendix is only available in electronic form at
} http://www. edpsciences.org made it possible to calibrate several secondary distance indicators (Freedman et al. 2001), which can be used to measure the distances to large numbers of more distant galaxies and to determine the global value of the Hubble constant.

At a given Cepheid period, the cosmic dispersion of the average absolute magnitude is so small $(\approx 0.2 \mathrm{mag})$ that the systematic errors in the measured distances to the calibrator galaxies were considered as negligible. Based on this premise, the analysis led several teams (including us) to almost concordant Cepheid distances (Freedman et al. 2001; Saha et al. 2001; Paturel et al. 2002b). The Hubble constant finally derived by the HST Key Project was $H_{0}=72 \pm 8 \mathrm{~km} \mathrm{~s}^{-1} \mathrm{Mpc}^{-1}$. It seemed to many that the dilemma between high and low $H$ was resolved, especially after the WMAP background radiation analysis led to a similar value. At the same time, the "precision" global cosmology from the WMAP and (future) PLANCK experiments has emphasized the growing importance of an accurate local $H$ determination to test the physics of the standard cosmological model (Spergel et al. 2003; Blanchard et al. 2003).

The distances from the HST data also confirmed that the Hubble law works at surprisingly small scales as observed in independent studies (Sandage \& Tammann 1975; Ekholm et al. 2001; Karachentsev et al. 2002). The Hubble diagram (velocity vs. distance) directly shows the local expansion rate $H$ as given by these remarkable HST observations. 


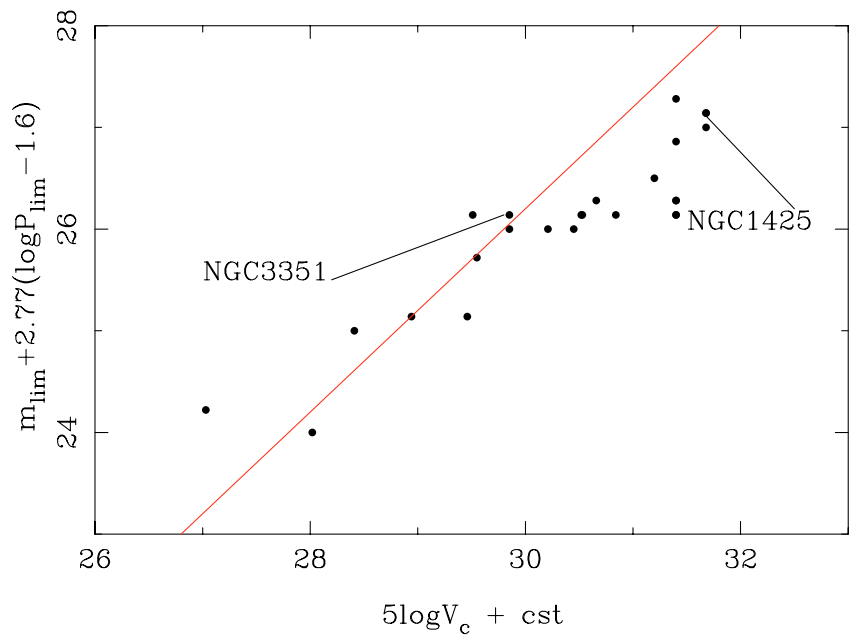

Fig. 1. Variation of the deepness of the Cepheid samples with distance. The (normalized) apparent $V$ magnitude limit of the Cepheid samples first grows with distance (radial velocity $V_{\mathrm{c}}$ ) but, after a certain point, tends to bend where we have seen the bias appearing in derived Cepheid distances. We show the positions for an unbiased galaxy (NGC 3351) and a biased one (NGC 1425).

\section{Evidence for the Cepheid bias}

We used this local Hubble law as an indicator of relative distances. The radial velocities, corrected for known velocity flows (our Galaxy moves with respect to the Local Group which moves towards the Virgo cluster) directly give relative distances without any assumption about $H$. It is then possible to test the behaviour of the measured HST distances against true relative distances. The conclusion is that they are affected by a new, significant bias (Teerikorpi \& Paturel 2002; henceforth TP02). Numerical simulations confirmed the familiar bias pattern (Paturel \& Teerikorpi 2004; henceforth PT04). The bias, not yet completely understood, is not due to the small scatter in the average PL-relation, but rather other factors are important such as the amplitude of variation of the magnitudes, the longest observable period and dust extinction, together with the cut-off effect of the limiting absolute magnitudes of the Cepheid sample (Sandage 1988) in $V$ and $I$-photometric bands, that are both needed for extinction corrections.

Figure 1 shows an important ingredient of the bias: how a relevant limiting magnitude of Cepheid samples changes with the kinematical distance. Ideally, one would like to see this limit increase in step with distance. But Fig. 1 reveals that the magnitude limit no longer grows properly above a certain distance. We have suggest that the biased region starts there for the HST distance determinations.

Another clue that suggests the presence of the bias, in addition to those already discussed in our previous papers, is given by Fig. 7a. The local Hubble diagram shows a departure from the expected linearity above a distance of $10 \mathrm{Mpc}$.

Finaly, it should be possible to see the departure from a proper sampling directly on the raw PL relation. As an example we show the $V$-band PL relation in Figs. $2 \mathrm{a}$ and $\mathrm{b}$, for an unbiased galaxy (NGC 3351) and a biased one (NGC 1425). The
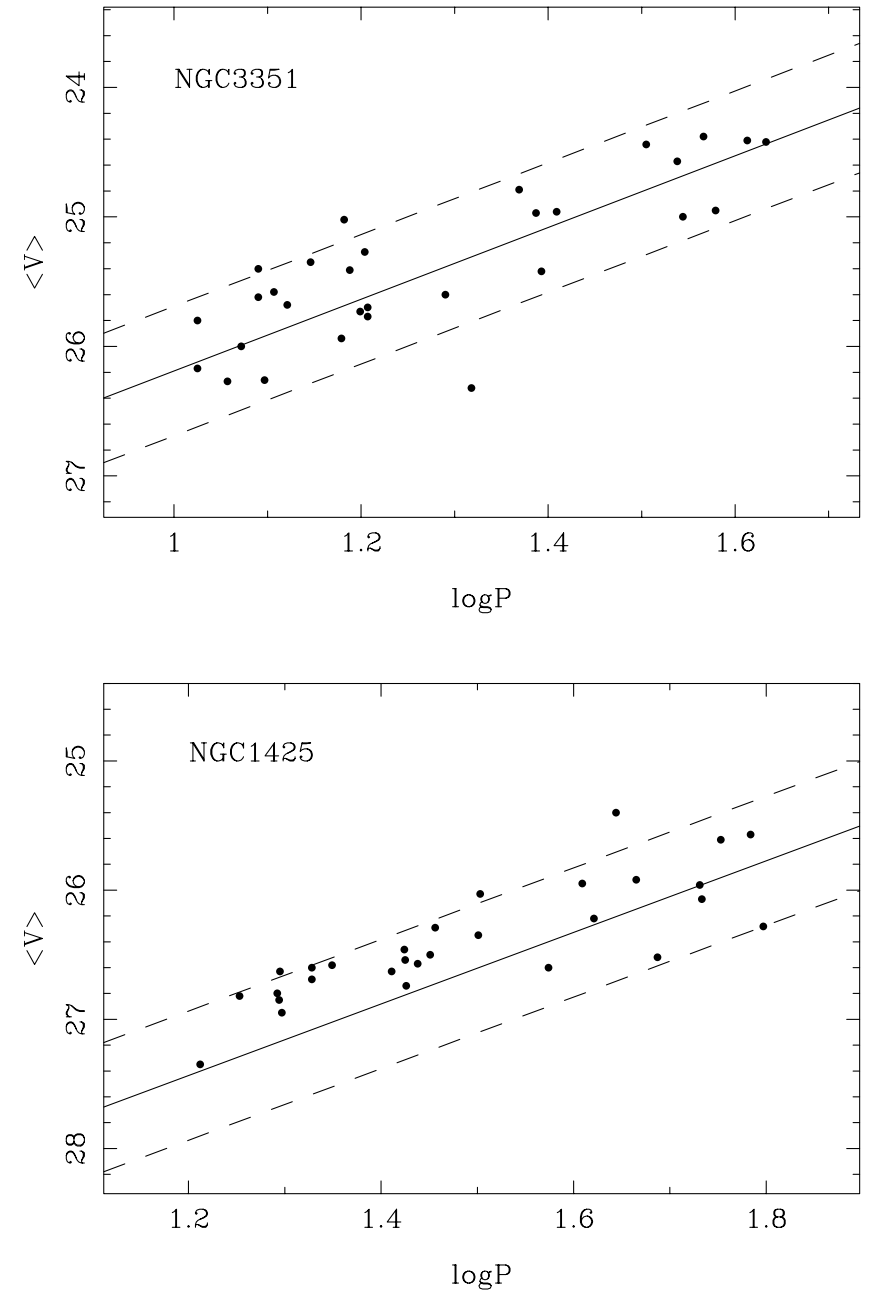

Fig. 2. $V$-band period luminosity relation for NGC 3351, an unbiased galaxy (top panel) and NGC 1425, a biased galaxy (bottom panel). These galaxies are shown in Fig. 6, where we compare the corrected and uncorrected distance moduli.

positions of these galaxies are shown in Figs. 1 and 6, where the bias effect is visible.

\section{Derivation of the bias correction curve}

How has this systematic error affected the cosmic distance scale, the local Hubble diagram and the value of the Hubble constant? We can apply a previous representation which is at the heart of traditional selection bias corrections. This was used several years ago by us to treat the analogous case of incompleteness bias in galaxy clusters (Teerikorpi 1987; Bottinelli et al. 1987). Although the Cepheid stars in a host galaxy are not totally equivalent to the galaxies themselves in a galaxy cluster as regards selection effects, the simulations show the typical bias pattern.

\subsection{Construction of the sample}

The calibrator galaxies with Cepheid distances are taken from the Hubble Space Telescope Key Project summary paper (Freedman et al. 2001) with their distance modulus $\mu$. We 
use radial velocities corrected for known peculiar motions towards the Local Group and the Virgo cluster (Yahil et al. 1977; Ekholm et al. 1999). We assigned a constant velocity to galaxies belonging to the same group (Leo, Fornax, Virgo and M 81-group). For the Virgo and Fornax clusters we adopted the cosmic velocities $1200 \mathrm{~km} \mathrm{~s}^{-1}$ and $1370 \mathrm{~km} \mathrm{~s}^{-1}$, respectively. The galaxies with corrected velocity below $150 \mathrm{~km} \mathrm{~s}^{-1}$ were not used for the construction of the bias curve to avoid the effect of random peculiar velocities. NGC 4414 was excluded because, at $18.9^{\circ}$ from the Virgo cluster, its corrected velocity is uncertain (Ekholm et al. 1999). The sample is given in the Appendix.

\subsection{The Hubble parameter and the magnitude limit}

From these data, we calculate the observed Hubble parameter for each calibrator $\log H=\log V_{\mathrm{c}}-0.2 \log \mu$ and their limiting absolute magnitude $M_{\mathrm{lim}}$. Similarly to TP02, this last quantity is calculated from the limiting apparent magnitude $m_{\text {lim }}$ of each calibrator (Paturel et al. 2002a) and a distance derived from the local Hubble law, with an arbitrary value of the Hubble parameter $(H=100)$, the choice of which does not influence this analysis. In Sect. 3.3. we also use the derived distance modulus $\mu$ to calculate another absolute limiting magnitude.

Thus, the absolute limiting magnitudes of the Cepheid samples are calculated either as:

$M_{\lim }\left(V_{\mathrm{c}}\right)=m_{\lim }-5 \log \left(V_{\mathrm{c}} / 100\right)-25$,

or as:

$M_{\lim }(\mu)=m_{\lim }-\mu$.

When $\log H$ is plotted against $M_{\lim }\left(V_{\mathrm{c}}\right)$, a trend appears (Fig. 3a) with a plateau near the bright $M_{\lim }\left(V_{\mathrm{c}}\right)$.

As in TP02, we then improve the $\log H-M_{\text {lim }}$ diagrams with a normalization of the absolute limiting magnitude. It is made using the list of limiting observational periods of Cepheid stars (TP02). Its rationale is to put all individual bias curves on the same mean curve by compressing or expanding the $x$-axis according to each individual period. This method highlights the phenomenon with less distortion, as shown by observations (TP02) and simulations (PT04).

The normalized absolute limiting magnitude is calculated as:

$M_{\mathrm{lim}}^{\mathrm{norm}}=M_{\mathrm{lim}}+2.77\left(\log P_{\mathrm{lim}}-1.6\right)$,

where $M_{\text {lim }}$ is calculated either from Eq. (1) or from Eq. (2). The adopted slope of the PL relation is 2.77 for $V$-band magnitudes. The normalized diagram is shown in Fig. $3 \mathrm{~b}$.

\subsection{A check for the correlation of errors}

In our first paper TP02 we realized that the trend in the parameter $\log H$ could be due to a correlation of errors. Indeed, in Fig. 3a, we calculate the limiting absolute magnitude from the radial velocity, according to Eq. (1). Thus, the radial velocity appears on both axes. If the observed velocity of a given object
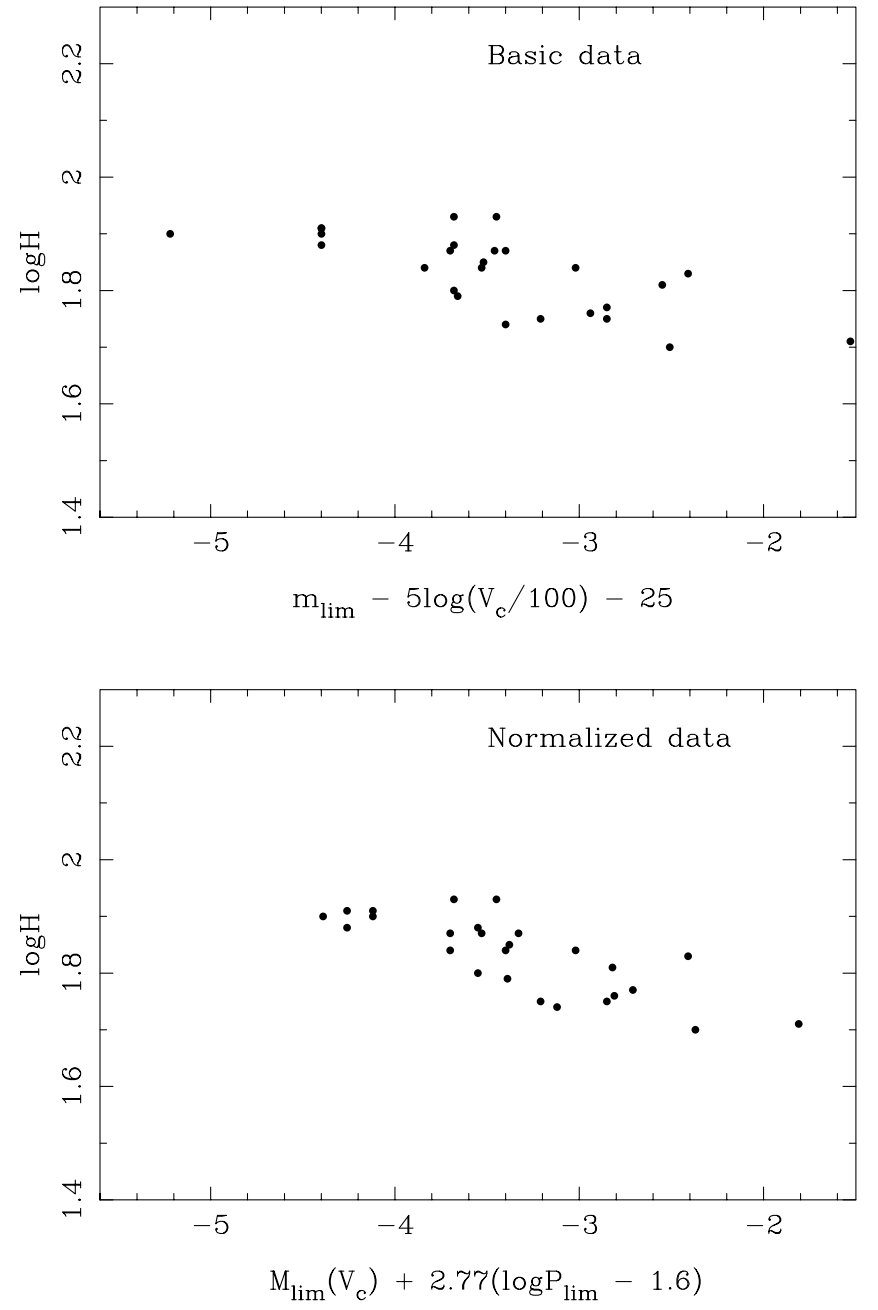

Fig. 3. Observed Hubble values in log scale against normal (top panel) and normalized (bottom panel) limiting absolute magnitude. These absolute magnitudes are calculated from Eq. (1), using corrected radial velocity $V_{\mathrm{c}}$. Top panel: the observed $\log H$ depends on the limiting absolute magnitude. One may recognize the classical shape of a bias curve with a plateau of constant $\log H$ near the faint limiting absolute magnitudes $\left(M_{\lim }>-3.4\right)$. Bottom panel: after the normalization, all the data are put on a same bias curve. The plateau is more clearly visible.

is too large, due, for instance, to random velocity, the corresponding point will be higher on the $y$-axis and lower on the $x$ axis. The point will be displaced in a direction with a negative slope. If the velocity is too small, the point will be displaced in the opposite direction, i.e., along the same line. This effect could thus produce something like the effect we have shown in Fig. 3a.

In TP02, we argued that the dispersion in the local Hubble law is small enough so that the systematic distance error dominates in the $\log H-M_{\mathrm{lim}}$ diagram. Indeed, at small distances, less than 5 to $10 \mathrm{Mpc}$, there is evidence for a low dispersion $\$ 50 \mathrm{~km} \mathrm{~s}^{-1}$ (e.g. Ekholm et al. 1999; Karachentsev et al. 2002, 2003; Thim et al. 2003), as we discussed in TP02. Also, we checked that the trend in the $\log H$ vs. $M_{\text {lim }}$ diagram is also visible for cluster members, when the cluster velocity is used for the members, which should be at the same (cluster) distance. 

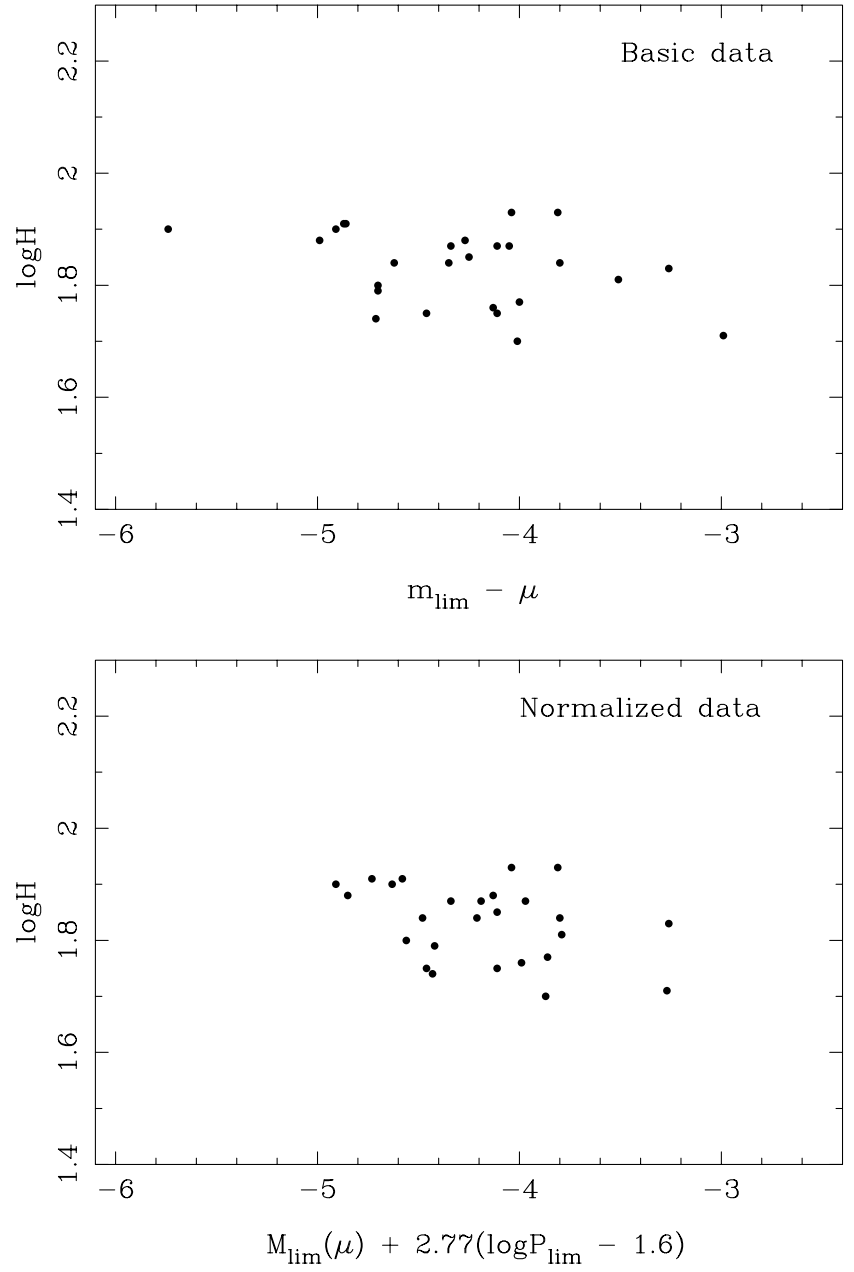

Fig. 4. Observed Hubble values in log scale against normal (top panel) and normalized (bottom panel) limiting absolute magnitude. These absolute magnitudes are calculated from Eq. (2), using observed distance moduli $\mu$. Top panel: the correlation of the observed $\log H$ with the limiting absolute magnitude is still visible, as in Fig. 3a, although it should be canceled by the inverse correlation of errors (see text). However it is difficult to recognize the classical shape of a bias curve because of the uncertainties on $\mu$. Bottom panel: after the normalization, all the data are put on the same bias curve.

If we repeat the test with $M_{\text {lim }}(\mu)$ (Eq. (2)), instead of $M_{\mathrm{lim}}\left(V_{\mathrm{c}}\right)$, there is also a correlation of errors but in the opposite direction. We thus expect a positive slope. The result is shown in Fig. 4a. There is no evidence of such a positive slope (on the contrary, the slope still seems slightly negative). The real trend is probably intermediate between these two extreme cases. If a true bias exists, its signature is expected to diminish in the $\log H$ vs. $M_{\text {lim }}$ diagram, when the kinematic distance is replaced by the biased photometric distance. Figure $4 \mathrm{~b}$ shows the same diagram, but with the normalization. Thus, we conclude that the observed trend may well be real. Because such a trend can result from a statistical bias (see the Appendix, and also PT04), we will apply the old precepts to analyze it, by fitting a classical bias curve.

\subsection{Fitting a bias curve}

The mathematical form of the bias curve $\Delta \log H$ vs. $M_{\lim }$ is taken from the incompleteness bias method (Teerikorpi 1975; 1987) normally used for cluster galaxies (Bottinelli et al. 1987). The form of the curve is fixed by two free parameters : a magnitude $(\langle M\rangle)$ and a dispersion $(\sigma)$. According to the simulations, $\sigma$ should take into account the amplitude of the variation of Cepheid stars.

$\Delta \log H=\sqrt{\frac{2 \sigma^{2}}{\pi}} \exp -\frac{a^{2}}{1+\operatorname{erf}(a)}$

with

$a=\frac{M_{\mathrm{lim}}-\langle M\rangle}{\sqrt{2 \sigma^{2}}} \quad \operatorname{erf}(x)=\frac{2}{\sqrt{\pi}} \int_{0}^{x} \mathrm{e}^{-t^{2}} \mathrm{~d} t$.

We adopted a dispersion $\sigma=0.6$, that approximately takes into account the amplitude of variation in the $V$-band for a typical Cepheid star oscillating in the fundamental mode. First, we estimated visually the parameters $\log H$ and $\langle M\rangle$ that reproduce the distribution of the observed points. We obtained $\log H=1.76$ and $\langle M\rangle=-4.8 \mathrm{mag}$. This result was confirmed from a fit on the data: for a series of $\log \tilde{H}$ values from 1.5 to 2.0 , with a step of 0.005 , and a series of $\langle M\rangle$ from -8 to -2 , with a step of 0.05 , we calculated the biased $\log H_{\text {bias }}=\log H+\Delta \log H$. Then we calculated the sum of the square of deviations:

$S=\sum_{\text {observ. }}\left(\log H_{\text {bias }}-\log \tilde{H}\right)^{2}$.

We kept as a result the $\log \tilde{H}$ and $\langle M\rangle$ values for the smallest $S$. The method was applied to the normalized data. The results are the following:

Fig. 3b: $\log H=1.76$ and $\langle M\rangle=-3.70$

Fig. 4b: $\log H=1.80$ and $\langle M\rangle=-4.95$.

The fit curves are shown in Figs. $5 \mathrm{a}$ and $\mathrm{b}$. The true values are somewhere within these limits, in agreement with our visual estimation. For the rest of the paper we will adopt our initial estimates: $\log H=1.76$ and $\langle M\rangle=-4.8$.

We emphasize that this adopted value of the parameter $\log H$ for the bias curve does not affect the global value of $H_{0}$, as derived in Sect. 5. Only the relative form $\Delta \log H$ of the bias curve is important there, giving an estimate of the distance bias for each calibrator galaxy.

We have added in Figs. 5a and b (filled star) the measurement of the galaxy M 83 (NGC5236) by Thim et al. (2003). They used the $8.2 \mathrm{~m}$ Unit Telescope 1 of the ESO VLT under subarcsecond seeing and could reach $m_{V} \approx 25$ and $P_{m} \approx 55 \mathrm{~d}$. This very good ground observation puts M 83 at $4.5 \mathrm{Mpc}$, directly in the unbiased plateau. This demonstrates the capability of the Very Large Telescope to measure Cepheid distances up to at least $5 \mathrm{Mpc}$.

In spite of the complexity of the bias, it is remarkable that the simple bias curve represents quite well the behaviour of real data. From the adopted bias curve we deduce the change $\Delta \log H$ and derive a new corrected distance for each calibrator galaxy. 

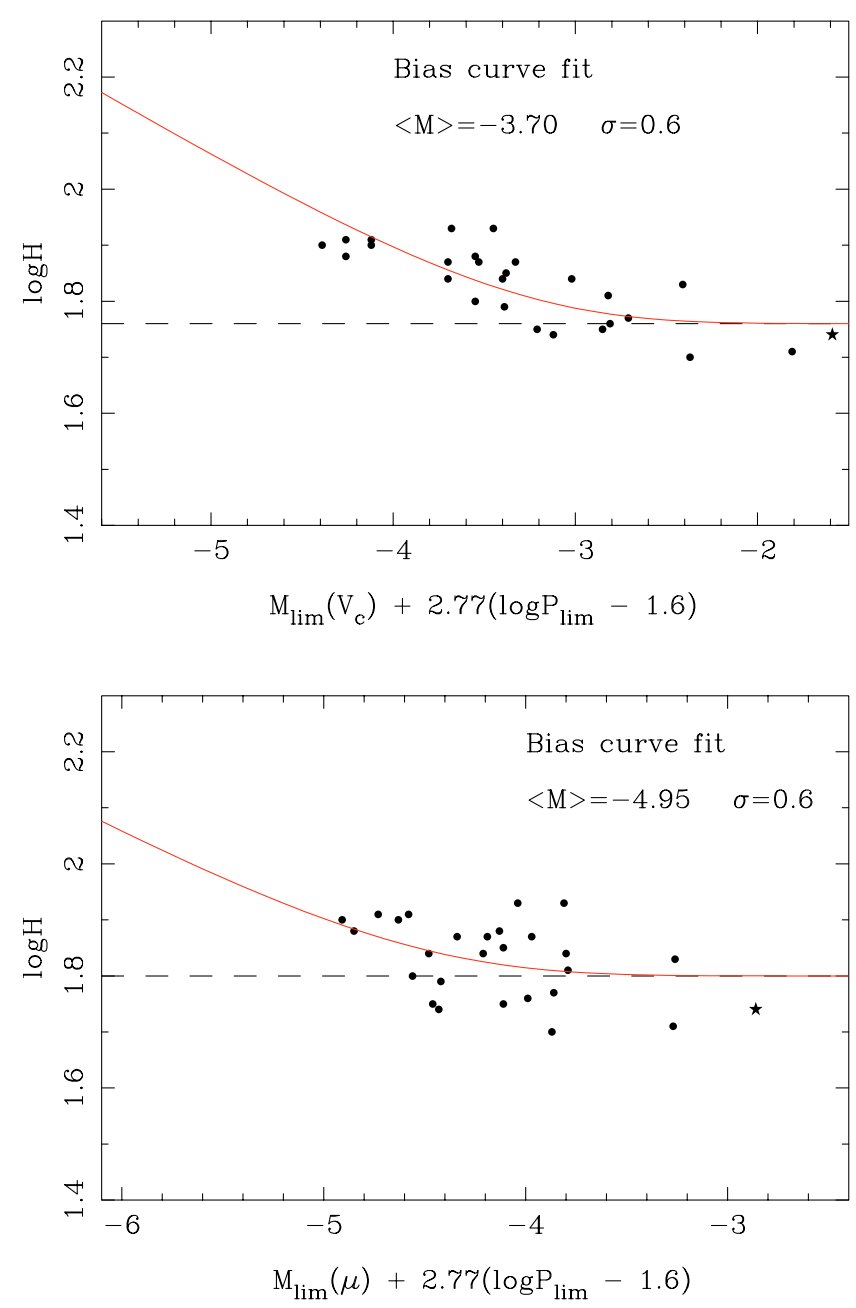

Fig. 5. Application of the cluster incompleteness bias curve fitting to the Cepheid bias. Top panel: the fit of Fig. $3 \mathrm{~b}$ with the bias curve following the method described in the text. Bottom panel: the same figure as the previous one, when the bias fit is applied to Fig. $4 \mathrm{~b}$. We have added on both figures the recent measurement of the galaxy M 83 (filled star) by Thim et al. (2003). It is in the unbiased plateau.

\section{Implications for the Local Hubble constant}

We add to the HST sample the previous ground-based Cepheid observations (Paturel et al. 2002b) (Fig. 6). The measurements from space and ground seem to be biased at different distances. The bias for ground-based data appears at a distance modulus $\mu \approx 25$ (i.e. $\approx 1 \mathrm{Mpc}$ ) while it starts at $\mu \approx 29.5$ (i.e. $\approx 8 \mathrm{Mpc}$ ) for the HST data, because the limiting magnitude of the HST is several magnitudes fainter.

Only about twenty percent of galaxies can be considered as unbiased. This seems surprising, but in fact the fraction is similar to typical cases of galaxy cluster incompleteness or field galaxy Malmquist biases (Bottinelli et al. 1987; Theureau et al. 1997). Fortunately, most of the biased galaxies are still very valuable when corrected.

The Hubble diagrams with uncorrected and corrected distances (Figs. 7a,b) show that the correction improves the linearity of the Hubble relation (the linear correlation coefficient increases from $0.966 \pm 0.012$ to $0.984 \pm 0.006$ ) and reduces the

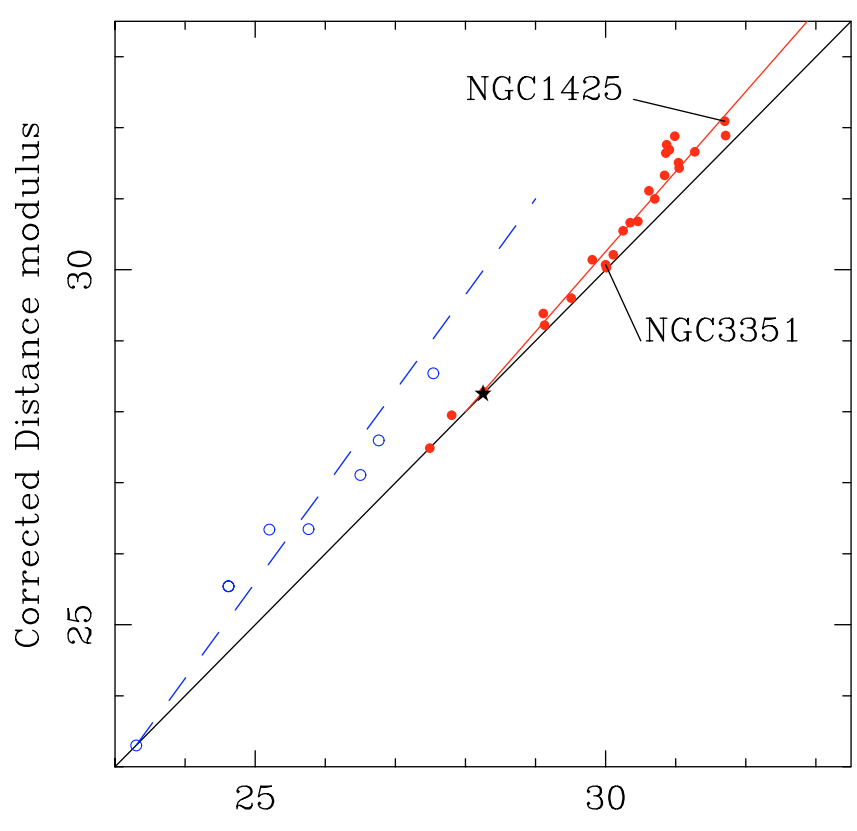

Uncorrected Distance modulus

Fig. 6. Comparison of corrected and uncorrected distance moduli for HST (light gray or red) and ground-based (dark gray or blue) observations. The ground-based (open circles) and HST observations (filled circles) show the same trend (dashed lines) at different distances as explained by the systematic error in Cepheid distances. From the ground, the bias starts soon beyond the closest galaxy (M31), except for the new VLT result on M 83 (filled star). From space, the bias appears beyond the distance of closest galaxy groups (M 81). At the distance of the closest large galaxy cluster (Virgo) it has a large incidence. The identified galaxies (NGC 3351 and NGC 1425) were used above (Fig. 2) to show the PL relation for an unbiased and biased galaxy.

dispersion (from 120 to $84 \mathrm{~km} \mathrm{~s}^{-1}$ ). The local value of $H_{\text {local }}$ drops from 69 to $56 \mathrm{~km} \mathrm{~s}^{-1} \mathrm{Mpc}^{-1}$. It was surprising in Freedman et al. (2001) that the local Hubble diagram for Cepheids was quite scattered. Now we propose that this was due to the variable distance bias.

Even in the nearer distance range, for galaxies observed from the ground with $V_{\mathrm{c}}<300 \mathrm{~km} \mathrm{~s}^{-1}$ (open circles in Fig. 7), the dispersion decreases from $35 \mathrm{~km} \mathrm{~s}^{-1}$ to $31 \mathrm{~km} \mathrm{~s}^{-1}$. These numbers again highlight the remarkable smoothness of the Hubble flow in the local inhomogeneous environment, for long recognized as a puzzle (e.g. Sandage 1999), but which has recently obtained theoretical clarification from $\Lambda \mathrm{CDM} N$-body simulations by Klypin et al. (2003) and Macciò et al. (2005), and other considerations by Teerikorpi et al. (2005). These studies support the suggestion by Chernin (2001) and Baryshev et al. (2001) that the low velocity dispersion may be a local signature of the dominating cosmological vacuum or dark energy.

\section{Implications for the global Hubble constant}

As these nearby galaxies were used for the calibration of five long-range distance criteria, the bias must have consequences on the determination of the global Hubble constant. Using 

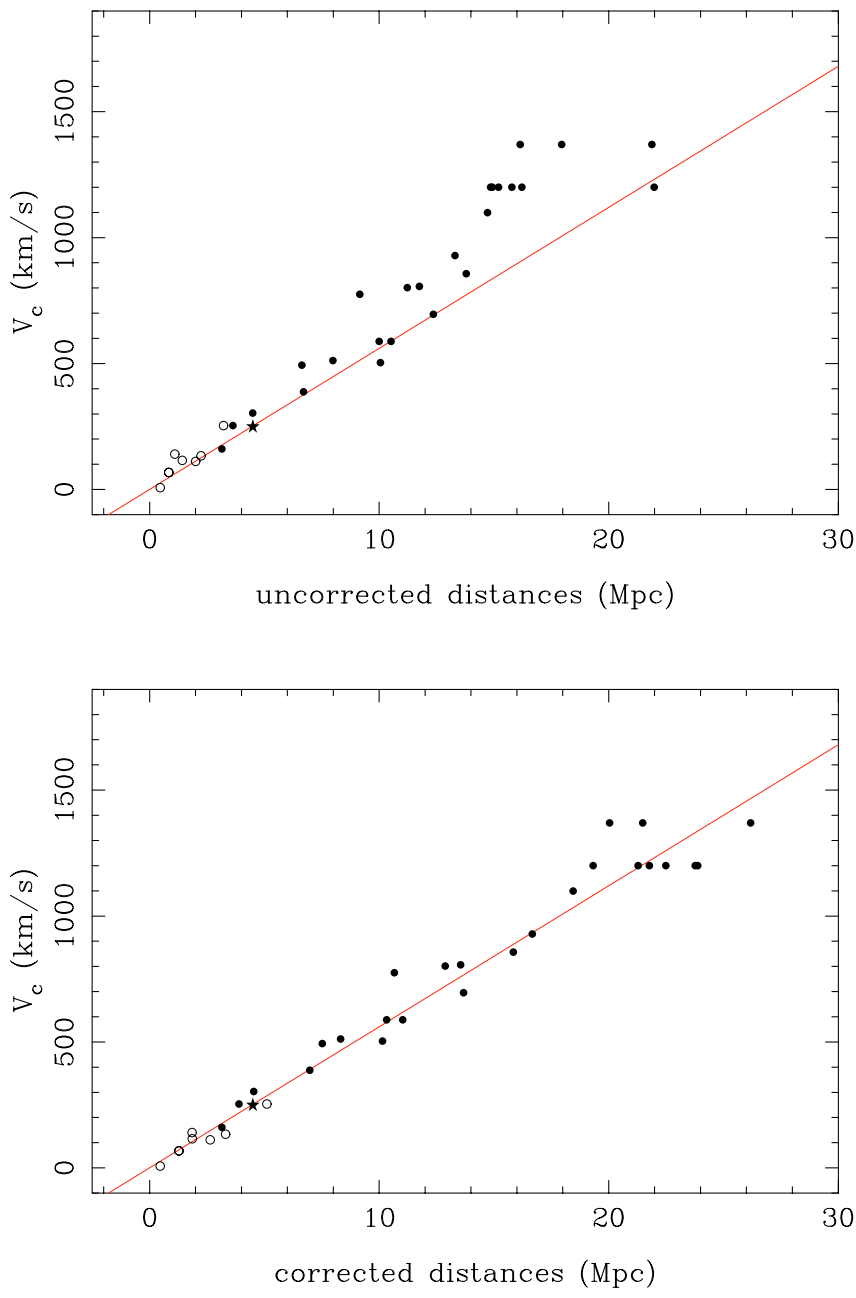

Fig. 7. Local Hubble diagrams for uncorrected (top) and corrected (bottom) distances. Top panel: the clear curvature in the uncorrected relation is now understood to be caused by the bias that moves the galaxies up from the Hubble line. Open circles show the galaxies observed from the ground with a velocity $V_{\mathrm{c}}<300 \mathrm{~km} \mathrm{~s}^{-1}$. Bottom panel: the linearity, which is the property of the large-scale Hubble law as shown by secondary distance indicators (e.g., supernovae Ia), is revealed here, locally, by Cepheid stars. The line corresponds to a local value $H=56 \mathrm{~km} \mathrm{~s}^{-1} \mathrm{Mpc}^{-1}$. The filled star represents the recent VLT measurement of the galaxy M 83 (see text).

Tables 2 and 12 in the HST summary paper (Freedman et al. 2001), it is possible to recalculate the new value of $H_{0}$ from each distance indicator.

Table 1 below summarizes the result. The value of $H_{0}$ from a given secondary distance indicator is calculated as a weighted mean $(w \propto N)$ of the contribution of each calibrator. The mean values of $H_{0}$ are calculated by weighting individual values according to the mean errors $\left(w \propto 1 / \sigma^{2}\right)$. Two important facts are seen: i) the range of individual values is reduced. In particular the Fundamental Plane method is no longer an enigmatic outlier, but is in good agreement with the two main distance indicators (SNIa and Tully-Fisher). ii) The Hubble constant is reduced by about 15 percent. The mean value from different distance indicators is $H_{0}=60 \mathrm{~km} \mathrm{~s}^{-1} \mathrm{Mpc}^{-1}$.
Table 1. Influence of the Cepheid bias on the Hubble constant from long-range distance criteria.

\begin{tabular}{lll}
\hline \hline Method & Uncorrected $H_{0}$ & Corrected $H_{0}$ \\
\hline SNIa & $71 \pm 2 \mathrm{~km} \mathrm{~s}^{-1} \mathrm{Mpc}^{-1}$ & $60 \mathrm{~km} \mathrm{~s}^{-1} \mathrm{Mpc}^{-1}$ \\
TF relation & $71 \pm 3$ & 58 \\
FP method & $82 \pm 6$ & 60 \\
SBF & $70 \pm 5$ & 61 \\
SNII & $72 \pm 9$ & 65 \\
\hline Weighted mean & $72 \pm 2.7$ & $60 \pm 1.3$ \\
\hline
\end{tabular}

$\dagger$ Based on the distances of Virgo, Fornax and Leo.

\section{Concluding remarks}

We do not present this result as a final one, but rather as indicative of the influence of the detected Cepheid bias on the cosmic distance scale. Firstly, we have used as uncorrected input values those $H_{0}$ derived by the HST Key Project. Further studies are needed on each long range distance criterion, all having their own specific problems. An example is the fact that the other HST programme on $H_{0}$ derived $H_{0}=60 \mathrm{~km} \mathrm{~s}^{-1} \mathrm{Mpc}^{-1}$ from the same supernova Ia data (Saha et al. 2001). Secondly, there are also uncertainties in the velocities of clusters and the memberships of their galaxies. For example, had we used the Key Project's preferred value for Virgo's velocity $\left(1350 \mathrm{~km} \mathrm{~s}^{-1}\right)$, the bias effect would have been still larger. Had we put some assumed Virgo galaxies in the foreground, this would have made $H_{0}$ from SNIa decrease less. We will study this question more fully elsewhere, but note here that the pure bias pattern in the $\Delta \log H$ vs. $M_{\text {lim }}$ diagram can be distorted or even amplified if an incorrect radial velocity is given to a galaxy. This may happen, for example, if a foreground or background galaxy is assigned to a cluster whose average velocity is then used for this galaxy. Thirdly, the studies by Tammann et al. (2003) and Sandage et al. (2004) on the non-uniqueness of the Cepheid PL-relation also emphasize the fact that the local distance ladder is not yet finally established.

How some astronomers (most notably A. Sandage and G. Tammann) in the pre-HST era, consistently derived values of $H_{0}$ of less than $60 \mathrm{~km} \mathrm{~s}^{-1} \mathrm{Mpc}^{-1}$, even though the number of calibrator galaxies was much smaller. With hindsight, we see that the distances to those nearby calibrators were less biased, while efforts were made to avoid the selection biases in field and cluster galaxy samples (Sandage \& Tamman 1975; Bottinelli et al. 1986).

We summarize the new results from this study:

- The bias correction used traditionally for cluster galaxies represents well the observed Cepheid star bias.

- HST and previous ground-based observations are both affected, showing the same trend which starts at difference distances.

- The recent VLT measurement of M 83 is unbiased, according to our criteria.

- After correction for the bias, the linearity of the local Hubble law is improved and the scatter is reduced.

- The distance scale has expanded by about 20 percent from the HST Key Project scale. 
- When the distances of the calibrator galaxies are corrected, the long-range distance criteria are in better agreement.

- Both local and global Hubble constants agree well and give $H$ around $58 \mathrm{~km} \mathrm{~s}^{-1} \mathrm{Mpc}^{-1}$, as based on the HST Key Project results.

Thus, attempts to use the observations at their extreme limit are generally hampered by hidden selection effects that might have been anticipated in the light of the history of cosmic distance measurements (Sandage 1995; Teerikorpi 1997). Nevertheless, the Hubble Space Telescope is making a large contribution in the local galaxy universe to establish the cosmic distance ladder. This relatively nearby region, where Edwin Hubble discovered his law, still has challenges. To observe Cepheid stars in the Virgo cluster and beyond in an unbiased manner would require a space telescope two or three times larger than the HST. Our work shows that even with the HST one can obtain Cepheid distances without the present bias problem if one confines the study to galaxies for which one can reach the normalized limiting magnitude fainter of at least -4 . Such galaxies would populate the unbiased plateau in the bias diagram (Fig. 5), allowing one to strengthen the basis of the cosmic distance scale in the distance range $1 \div 10 \mathrm{Mpc}$, which is also kinematically and dynamically a very interesting region (cf. Thim et al. 1997).

Acknowledgements. This work has been supported by the Academy of Finland (the project "Fundamental questions of observational cosmology"). We thank G. Tammann for proposing a test to see the influence of the correlation of errors. We thank the referee for reading the manuscript with extreme attention.

\section{References}

Baryshev, Yu., Chernin, A., \& Teerikorpi, P. 2001, A\&A, 378, 279 Blanchard, A., Douspis, M., Rowan-Robinson, M., \& Sarkar, S. 2003, A\&A, 412, 35

Bottinelli, L., Gouguenheim, L., Paturel, G., \& Teerikorpi, P. 1986, A\&A, 156, 157
Bottinelli, L., Fouqué, P., Gouguenheim, L., Paturel, G., \& Teerikorpi 1987, A\&A, 181, 1

Chernin, A. 2001, Physics-Uspekhi, 44, 1153

de Vaucouleurs, G. 1984, The Observatory 102, No. 1050, p. 178

Ekholm, T., Lanoix, P., Teerikorpi, P., Paturel, G., \& Fouqué, P. 1999, A\&A, 351, 827

Ekholm, T., Baryshev, Yu., Teerikorpi, P., Hanski, M. O., \& Paturel, G. 2001, A\&A, 368, L17

Freedman, W. L., Madore, B. F., Gibson, B. K., et al. 2001, ApJ, 553, 47

Karachentsev, I. D., Sharina, M. E., Makarov, D. I., et al. 2002, A\&A, 389,812

Karachentsev, I. D., Makarov, D. I., Sharina, M. E., et al. 2003, A\&A, 398,479

Kennicutt, R. C. Jr, Freedman, W. L., \& Mould, J. R. 1995, AJ, 100, 1476

Klypin, A., Gottlöber, S., Kravtsov, A. V., \& Khoklov, A. M. 2003, ApJ, 596, 19

Leavitt, H. S., \& Pickering, E. C. 1912, Harvard College Observ. Circ., 173, p. 1

Macciò, A., Governato, F., \& Horellou, C. 2005, MNRAS, 359, 941

Madore, B. F., \& Freedman, W. L. 1991, PASP, 103, 933

Paturel, G., Theureau, G., Fouqué, P., et al. 2002a, A\&A, 383, 398

Paturel, G., Teerikorpi, P., Theureau, G., et al. 2002b, A\&A, 389, 19

Paturel, G., \& Teerikorpi, P. 2004, A\&A, 413, L31

Saha, A., Sandage, A., Tammann, G. A., et al. 2001, ApJ, 562, 314

Sandage, A. 1988, Publ. Astron. Soc. Pac., 100, 935

Sandage, A. 1995, in The deep universe, ed. Binggeli, \& R. Buser (Berlin: Springer-Verlag), p. 1

Sandage, A. 1999, ApJ, 527, 479

Sandage, A., \& Tammann, G. A. 1975, ApJ, 196, 313

Sandage, A., Tammann, G. A., \& Reindl, B. 2004, A\&A, 424, 43

Spergel, D. N., Verde, L., Peiris, H. V., et al. 2003, ApJS, 148, 175

Tammann, G. A. 1996, PASP, 108, 1083

Tammann, G. A., Sandage, A., \& Reindl, B. 2003, A\&A, 404, 423

Teerikorpi, P. 1975, A\&A, 45, 117

Teerikorpi, P. 1987, A\&A, 173, 39

Teerikorpi, P. 1997, ARA\&A, 35, 101

Teerikorpi, P., Chernin, A., \& Baryshev, Yu. 2005, A\&A, 440, 791

Teerikorpi, P., \& Paturel, G. 2002, A\&A, 381, L37

Theureau, G., Hanski, M., Ekholm, T., et al. 1997, A\&A, 322, 730

Thim, F., Tammann, G. A., Saha, A., et al. 2003, ApJ, 590, 256

Yahil, A., Tammann, G. A., \& Sandage, A. 1977, AJ, 217, 903 
G. Paturel and P. Teerikorpi: The extragalactic Cepheid bias, Online Material p 1

\section{Online Material}


G. Paturel and P. Teerikorpi: The extragalactic Cepheid bias, Online Material p 2

Table A.1. The sample used in this paper.

\begin{tabular}{lrrrr}
\hline \hline galaxy & $\mu_{\text {HSTKP }}$ & $m_{\lim }$ & $\log P_{\lim }$ & $V_{\mathrm{c}}$ \\
\hline IC 4182 & 28.26 & 25.0 & 1.60 & 303. \\
NGC 1326A & 31.04 & 27.0 & 1.60 & 1370. \\
NGC 1365 & 31.27 & 27.0 & 1.65 & 1370. \\
NGC 1425 & 31.70 & 27.0 & 1.65 & 1370. \\
NGC 2090 & 30.35 & 26.0 & 1.65 & 807. \\
NGC 2541 & 30.25 & 26.0 & 1.65 & 802. \\
NGC 3031 & 27.80 & 24.0 & 1.60 & 254. \\
NGC 2403 & 27.54 & 21.8 & 1.90 & 254. \\
NGC 3198 & 30.70 & 26.0 & 1.70 & 857. \\
NGC 3319 & 30.62 & 26.0 & 1.65 & 929. \\
NGC 3351 & 30.00 & 26.0 & 1.65 & 588. \\
NGC 3368 & 30.11 & 26.0 & 1.60 & 588. \\
NGC 3621 & 29.11 & 25.0 & 1.65 & 493. \\
NGC 3627 & 30.01 & 26.0 & 1.65 & 504. \\
NGC 4258 & 29.51 & 26.0 & 1.50 & 512. \\
NGC 4321 & 30.91 & 26.0 & 1.70 & 1200. \\
NGC 4496A & 30.86 & 26.0 & 1.70 & 1200. \\
NGC 4535 & 30.99 & 26.0 & 1.65 & 1200. \\
NGC 4536 & 30.87 & 26.0 & 1.65 & 1200. \\
NGC 4548 & 31.05 & 27.0 & 1.55 & 1200. \\
NGC 4639 & 31.71 & 27.0 & 1.70 & 1200. \\
NGC 4725 & 30.46 & 26.0 & 1.60 & 696. \\
NGC 5253 & 27.49 & 24.5 & 1.50 & 161. \\
NGC 5457 & 29.13 & 25.0 & 1.65 & 388. \\
NGC 7331 & 30.84 & 26.5 & 1.60 & 1099. \\
NGC 925 & 29.81 & 26.0 & 1.60 & 775. \\
\hline & & & &
\end{tabular}

\section{Appendix A: Reality of the Cepheid bias from a simple simulation}

In our second paper (Paturel \& Teerikorpi 2004), we analyzed the bias using detailed simulations. Here, we will repeat the simulations using a very simple method. We constructed an artificial Cepheid sample for the real galaxies below, with 200 Cepheids for each galaxy.

The true distances are derived from the radial velocity and an arbitrary Hubble constant. Then, from a Gaussian distribution $(G(1 ., 0.5))$ of $\log P$,we obtain the absolute magnitudes through realistic $V$ - and $I$-band PL relations. Then, from distances and absolute magnitudes we obtain apparent magnitudes $V$ and $I$. Each quantity is combined with realistic errors and an extinction is added to the apparent magnitudes from random color excess and proper extinction coefficients. Thus we constructed for true galaxies an artificial catalog of Cepheids with known periods and apparent magnitudes, like a real one. However, for these data we know the true distances and realistic observational limits for periods and apparent magntiudes.

Thus, making classical derivations of distances from the $V$ and $I$ Period-luminosity relations, we construct the bias diagram (as in Fig. 3), for two cases:

1) without any restriction on the Cepheids, as if they could all be observed (Fig. A.1a);

2) using only those Cepheids brighter than a limiting magnitude: for each galaxy, only the Cepheids brighter than
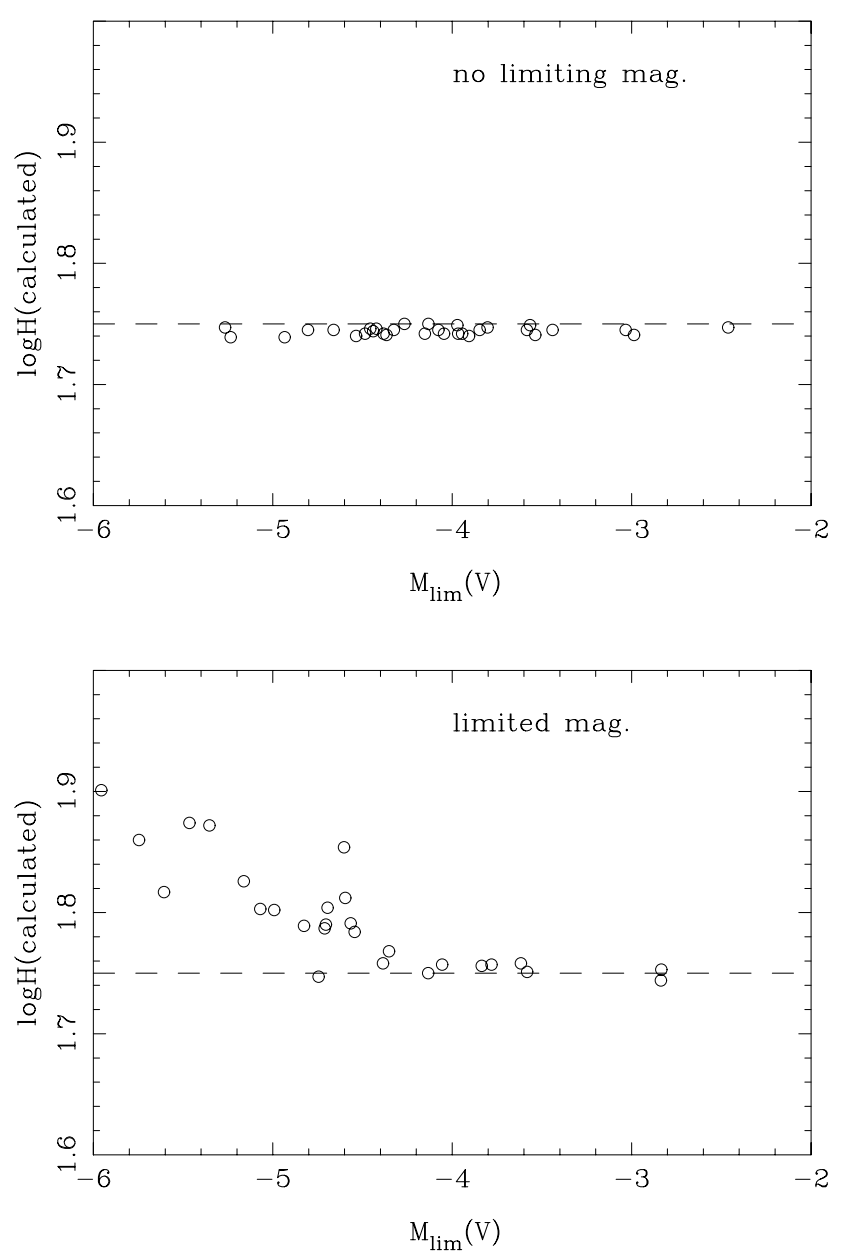

Fig. A.1. Simulation of the bias. Top panel: calculation of $\log H$ from a simulation, in the case where there is no selection effect on magnitudes. Bottom panel: the same calculation as above, but a with a realistic selection on magnitudes (the limiting magnitude includes the amplitude of the Cepheid light curve).

$m_{\mathrm{lim}}-\Delta m$ are kept for the calculation (here $m_{\mathrm{lim}}$ is a realistic limiting magnitude of Cepheids for the considered galaxy and $\Delta m$ is their amplitude) (Fig. A.1b).

The expected result is confirmed. This shows that the bias can be explained by a selection effect on magnitudes.

To see the effect of the velocity dispersion, we added to the simulated galaxies a random velocity dispersion increasing with distance, in order to reproduce the observations that show a very small velocity dispersion in the nearby universe. The adopted velocity dispersion is: $\sigma(V)=0.1 \mathrm{~V}+20\left(\right.$ in $\mathrm{km} \mathrm{s}^{-1}$ ). We obtained Fig. A.2a, b that reproduce quite well Figs. 5a, b based on real data. This also confirms that the bias is visible, even when it should be cancelled by an inverse correlation of errors (Bottom panel). The velocity dispersion contributes to hide the bias. The bias being visible with real data, this suggests again that the local velocity dispersion is very small. 
G. Paturel and P. Teerikorpi: The extragalactic Cepheid bias, Online Material p 3
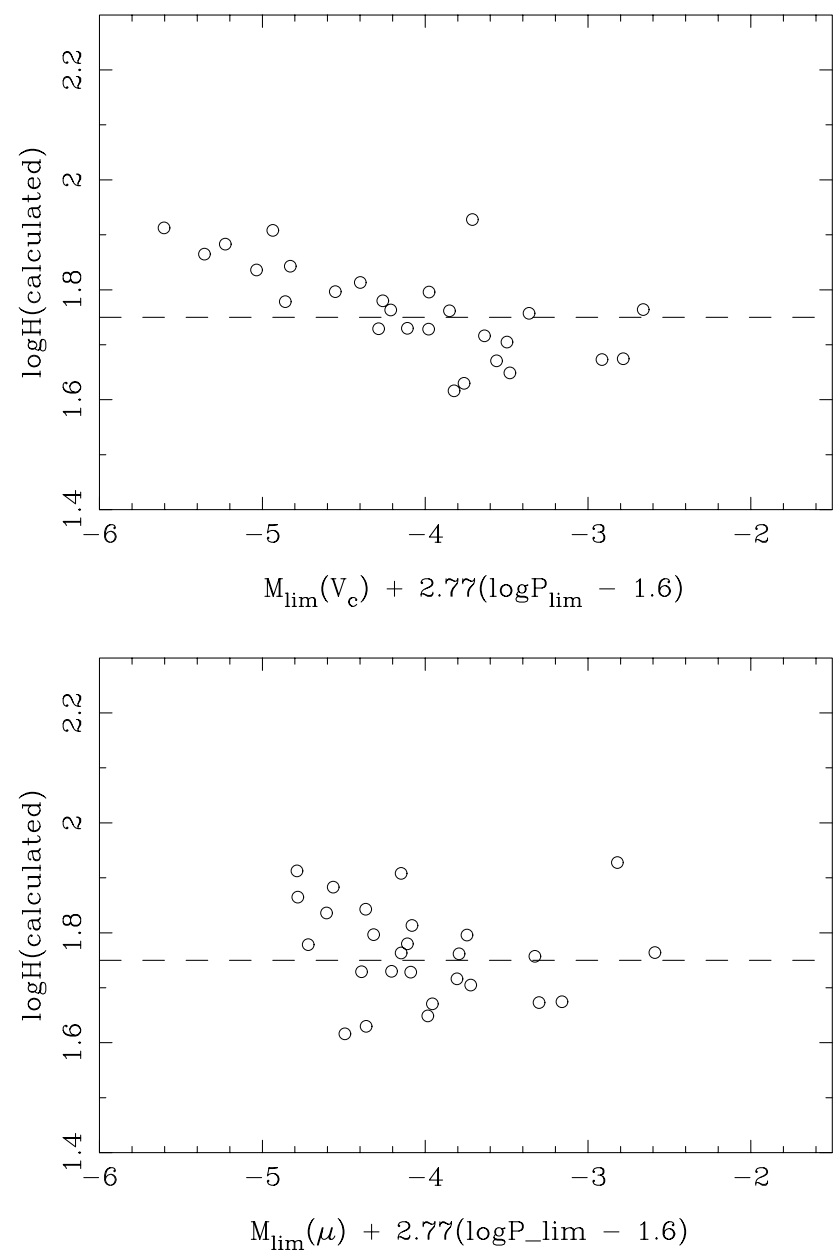

Fig. A.2. Simulation of the bias when we add a velocity dispersion increasing with distance. Top panel: case where the correlation of errors due to the velocity reinforces the bias trend. Bottom panel: case where the correlation of errors due to $\mu$ should produce a trend opposite to the trend produced by the bias. 